Revue d'histoire de l'enfance « irrégulière »

Le Temps de l'histoire

9 | 2007

Violences et jeunesse

\title{
Violence et internat : les centres de rééducation pour filles, en France, de la Libération au début des années 1960
}

Anne Thomazeau

\section{(2) OpenEdition \\ Journals}

Édition électronique

URL : http://journals.openedition.org/rhei/2163

DOI : 10.4000/rhei.2163

ISBN : 978-2-7535-1648-9

ISSN : $1777-540 \mathrm{X}$

Éditeur

Presses universitaires de Rennes

Édition imprimée

Date de publication : 15 novembre 2007

Pagination : 107-125

ISSN : 1287-2431

Référence électronique

Anne Thomazeau, « Violence et internat : les centres de rééducation pour filles, en France, de la

Libération au début des années 1960 », Revue d'histoire de l'enfance «irrégulière » [En ligne], 9 | 2007,

mis en ligne le 01 novembre 2009, consulté le 03 décembre 2020. URL : http://

journals.openedition.org/rhei/2163; DOI : https://doi.org/10.4000/rhei.2163 


\section{Violence en internat :}

les centres de rééducation pour filles, en France, de la Libération au

\section{début des années 1960}

Le 6 mai 1947, à Fresnes, « dans les bâtiments affectés aux filles dépendant de l'Éducation surveillée ", des mineures « escaladent le mur et tournent dans le chemin de ronde en chantant. Elles s'arment de haches, de marteaux, de barres de fer, elles cherchent du pétrole pour mettre le feu ». Une fois le calme rétabli, grâce à des renforts de police, " quarante détenues sont appréhendées et envoyées au dépôt de la préfecture de police. Là, certaines filles se révoltent à nouveau, démolissent les lits, brisent les vitres et défoncent les portes $»{ }^{(2)}$ Le 31 octobre 1950, à Cadillac, établissement d'éducation surveillée, Marguerite B., 19 ans, est retrouvée pendue dans sa cellule, après avoir plusieurs fois alerté l'institution en s'automutilant. ${ }^{(3)}$

Mettre en exergue ces deux événements ne signifie pas qu'ils sont le quotidien des internats de filles d'après-guerre. Au contraire, ils font plutôt figure d'exception - non par leurs formes assez ordinaires (violence collective et violence sur soi) mais par leur ampleur -, voire de contre modèles parce qu'ils concernent deux établissements marqués par leur passé carcéral, alors même que l'ambition de l'Éducation surveillée est, à partir des années 1940, de renouveler ses méthodes. ${ }^{(4)}$ Dans un esprit de réforme, illustré notamment par l'ordonnance de 1945 sur l'enfance délinquante affirmant le primat de l'éducation sur la répression, les acteurs du secteur tentent de mettre en place un modèle innovant de prise en charge, rompant avec le passé pénitentiaire et l'héritage des maisons de correction, de redressement et de relèvement : l'internat de rééducation. ${ }^{(5)}$

Pour autant, ce nouveau modèle de prise en charge ne paraît pas exempt de violence, surtout si on s'intéresse non seulement à ses rares manifestations spectaculaires, mais aussi à ses formes plus discrètes et ordinaires, c'est-à-dire l'ensemble des comportements et des actes ressentis comme violents. En
Anne

Thomazeau $^{(1)}$
(1) Professeur agrégée

d'histoire-géographie.

Préparation d'un doctorat d'histoire contemporaine sur la rééducation en internat des jeunes filles délinquantes et inadaptées, de la Libération aux années 1970 , sous

la direction d'Anne-Marie Sohn et de Jean-Noël Luc.

(2) Françoise Tétard, «Fresnes 1947, la révolte des inéducables ", les Cahiers de Vaucresson, $\mathrm{n}^{\circ} 2,1982$, p. 7-8.

(3) Béatrice Koeppel, Marguerite B., une jeune fille en maison de correction, Paris, Hachette, 1987, p. 7.

(4) Cadillac est vite considéré comme inadapté

Anne Thomazeau / p. 107 à 125 
et ferme en 1952, suite notamment à ce suicide.

(5) L'internat de rééducation n'est pas le seul mode de prise en charge existant dans la période étudiée. D'autres structures commencent alors à se développer comme les foyers de semi-liberté et les prises en charge en milieu ouvert. Toutefois, cette étude se limite aux internats de rééducation et, en particulier, aux internats de "grandes ", c'est-à-dire d'environ 14 à 21 ans.

(6) François Dubet, «A propos de la violence et des jeunes", Cultures et conflits, $\mathrm{n}^{\circ} 6$, été 1992, p. 7.

(7) Ainsi, deux mémoires de religieuses du Bon Pasteur d'Angers portent spécifiquement sur la " révolte juvénile ".

(8) «Quelques conseils à méditer et... à relire de considérant que, comme le souligne François Dubet, « la violence est définie par sa représentation, parce qu'elle n'est rien d'autre que ce qui est vécu comme une violence dans une culture, dans un groupe et dans un contexte d'interaction ", ${ }^{(6)}$ nous tenterons de repérer et d'analyser la violence des mineures - c'est-à-dire leurs comportements qualifiés de violents par l'institution -, la violence éventuelle de l'institution - les actes qui peuvent être ressentis par les élèves comme de la violence - et, enfin, les rapports et les interactions entre ces deux catégories de violence.

Lorsqu'elle émane des filles, la violence est perceptible dans les témoignages, dans les mémoires rédigés pour l'obtention du diplôme d'éducatrice et surtout dans les dossiers individuels des mineures placées. ${ }^{(7)}$ Ces derniers sont, en effet, très riches grâce aux nombreux écrits rédigés par les éducatrices sur les mineures et conservés dans les dossiers. À l'institution publique de Brécourt, par exemple, les éducatrices doivent "remettre le jour même à Madame la Sous-Directrice un rapport écrit sur tout incident sérieux et sur tout fait significatif ». ${ }^{(8)}$ À travers ces différentes sources, la violence des filles se révèle protéiforme autant dans ses manifestations - révoltes collectives, agressions contre le personnel, dégradations de matériel, tentatives de suicide, etc. - que dans ses motivations ou explications - " elle peut être [...] organisée, imprévisible, instrumentale, "irrationnelle" ». ${ }^{(9)}$ Cependant, un type de violence semble plus particulièrement retenir l'attention des acteurs, sans doute car il est le plus facilement repérable et identifiable : la violence physique, celle qui atteint l'intégrité des biens ou des personnes.

L'éventuelle violence de l'institution est plus difficile à saisir, peut-être justement parce qu'elle ne s'inscrit pas dans une définition étroite de la violence comme violence physique, celle-ci étant proscrite. Cependant, quelques éléments apparaissent en creux dans les dossiers individuels, lorsque, par exemple, la sanction reçue est mentionnée (ainsi que la réaction de la mineure), ou encore dans certains documents qui légitiment le recours à une méthode coercitive. Ainsi, en 1967, une religieuse consacre son mémoire de fin d'études d'éducatrice au "rôle préventif et curatif de l'isolement ». ${ }^{(10)}$

Dans un premier temps, nous verrons que la volonté de réforme et les profonds changements qui en découlent ne paraissent pas impliquer, aux yeux des acteurs du secteur, une disparition de la violence dans les internats de 
rééducation. Au contraire, une certaine violence des jeunes filles paraît normale et est attendue comme un mal logique dont il faut tenter de prévenir les symptômes, et ce bien que la grande majorité des jeunes filles ne soit pas placée pour des faits de violence. Il conviendra alors de se demander dans quelle mesure cette attente est liée à de réelles manifestations de violence. Dans cet objectif, nous tenterons de présenter les différentes formes de la violence des filles en internat tout en cherchant à saisir, d'une part, la réaction de l'internat et, d'autre part, l'articulation entre la violence des mineures et les modalités de la rééducation.

\section{Une violence attendue}

À en croire les acteurs du secteur, la violence des élèves est un mal attendu dans un internat de rééducation de filles. Pourtant, rares sont les mineures placées suite à des actes de violence.

\subsection{Les raisons du placement : rarement un comportement violent}

Les délinquantes représentent toujours moins de la moitié des mineures placées dans les internats de rééducation, et cette proportion diminue au cours de la période : de près de $40 \%$ en 1952 à 13,5 \% en 1961.(11) Même à l'institut professionnel d'éducation surveillée (IPES) de Brécourt, établissement public supposé accueillir les plus difficiles, les délinquantes sont minoritaires par rapport aux jeunes filles placées par mesure de protection.

\section{Origine judiciaire des mineures entrées à Brécourt}

\begin{tabular}{|c|c|c|c|c|c|c|}
\hline $\begin{array}{c}\text { Année } \\
\text { d'entrée }\end{array}$ & $\begin{array}{c}\text { Délin- } \\
\text { quance } \\
\text { Ordonnance } \\
\text { de 1945 }\end{array}$ & $\begin{array}{c}\text { Vagabon- } \\
\text { dage }\end{array}$ & $\begin{array}{c}\text { Correction } \\
\text { paternelle }\end{array}$ & $\begin{array}{c}\text { Assistance } \\
\text { éducative } \\
\text { Ordonnance } \\
\text { de 1958 }\end{array}$ & $\begin{array}{c}\text { Autres } \\
\text { ou } \\
\text { non précisé }\end{array}$ & $\begin{array}{c}\text { Total } \\
\text { des } \\
\text { entrées }\end{array}$ \\
\hline 1947 & 9 & 9 & 3 & - & 7 & 28 \\
1952 & 8 & 4 & 7 & - & 0 & 19 \\
\hline 1962 & 6 & - & - & 16 & 0 & 22 \\
\hline
\end{tabular}

temps à autre ", établissement de Brécourt, Centre des archives contemporaines, Fontainebleau, 2000 0111-8. Dossiers des mineures, Brécourt, 688 W 1 à 526, Archives départementales du Vald'Oise. La plus grande prudence est de rigueur pour utiliser ces dossiers individuels, et ce d'autant plus que leur étude n'est pas achevée. L'échantillon étudié n'est pour l'instant constitué que de 69 dossiers, ceux des mineures entrées dans l'établissement au cours de trois années (fin des années 1940, début des années 1950, début des années 1960). Les chiffres obtenus ne sont donc qu'indicatifs.

(9) François Dubet, op. cit., p. 8.

(10) Søur Dublanc, « L'isolement, rôle préventif et curatif de l'isolement en centre d'observation pour adolescentes inadap- 
tées ", Cahiers psychopédagogiques, $\mathrm{n}^{\circ} 54$, juillet 1967 , p. 4- 25.

(11) Rapport annuel de la direction de l'Éducation surveillée, 1962, p. 197.

(12) Résumé du cas, dossier individuel de la mineure H 27 , Brécourt, archives départementales du Vald'Oise, 688 W 271.

(13) Éric Pierre et David Niget, «Filles et garçons devant le tribunal des enfants et adolescents d'Angers de 1914 à 1940. Un traitement différencié ", in Christine Bard et al., [dir.], Femmes et justice pénale, XIXèmeXXème siècles, Rennes, Presses universitaires de Rennes, 2002, p. 333.

(14) Monique Néry, Synthèse de l'ensemble des informations contenues dans les différentes recherches déjà conduites à
De plus, la plupart des jeunes filles placées pour délinquance le sont à la suite de vols ou, moins souvent, d'outrages publics à la pudeur, et non d'un comportement violent. Ainsi, à Brécourt, parmi les vingt-trois mineures placées au titre de l'ordonnance de 1945, dix-sept jeunes filles sont accusées de vols (parfois assortis d'autres délits comme le recel), quatre d'outrages à la pudeur ou d'attentats aux mœurs, une de tentative d'avortement et, enfin, une d'infanticide associé à un outrage public à la pudeur. En outre, certaines jeunes filles accusées de vols, ou même, pour l'une d'elles, de "vandalisme en bande ", ${ }^{(12)}$ sont, en fait, placées au titre de mesures de protection. Ces dernières - vagabondage, qui est encore, après la seconde guerre mondiale, une " catégorie juridique fourre-tout ", ${ }^{(13)}$ correction paternelle, assistance éducative à partir de l'ordonnance de 1958 - recouvrent, en effet, des situations très diverses, même s'il s'agit souvent de contrôler le comportement sexuel des jeunes filles. Comme le souligne Monique Néry, « une même incrimination peut recouvrir des conduites très différentes, une même conduite peut être saisie sous plusieurs incriminations ».(14)

De ce fait, pour repérer les filles qui auraient commis des actes de violence, le plus pertinent est de dépasser l'analyse en termes de catégories judiciaires et de chercher au sein des dossiers individuels le détail des faits qui ont conduit au placement, tels qu'ils sont rapportés par les différents intervenants sociaux. Ainsi, parmi les soixante-neuf filles étudiées à Brécourt, seulement cinq se voient explicitement reprocher des actes de violence : un infanticide, des coups et un comportement violent pour deux, du "vandalisme en bande " pour une autre, et enfin une rébellion à Chevilly-Larue, où elle avait été placée au titre du vagabondage, pour une dernière. Peut-être faut-il ajouter Marcelle à qui sont reprochés les faits suivants : "Se croyant enceinte des œuvres d'un garçon de cirque, la mineure prit des pilules et a sauté d'un arbre pour essayer de se faire avorter. " (15)

Rares sont donc les placements résultant d'une violence exercée par la mineure. En revanche, plusieurs catégories peuvent dissimuler une violence subie. Ainsi, l'incrimination d'outrage public à la pudeur peut recouvrir des situations variées, jusqu'à une sexualité incestueuse imposée. C'est le cas pour Bernadette, également inculpée d'infanticide pour avoir tué le fruit de ces relations contraintes avec son beau-père. ${ }^{(16)}$ De même, les différentes mesures 
de protection des mineur(e)s peuvent concerner des filles victimes de maltraitance, de violences sexuelles, d'inceste, etc. Ainsi, dans l'échantillon étudié, la maltraitance est avérée pour trois mineures et trois jeunes filles ont été victimes de viols.

Il apparaît donc que, lorsque la violence figure dans les motivations du placement, il s'agit au moins autant de violence subie que de violence exercée. Pourtant, aussi bien dans les mémoires de religieuses du Bon Pasteur que dans les écrits de la directrice de l'établissement public de Brécourt, un certain nombre de manifestations violentes au sein des internats est présenté comme « normal».

\subsection{Une violence logique}

Le caractère logique et inéluctable de la violence en internat est expliqué par plusieurs facteurs, dont le premier semble être l'âge des jeunes filles, qui ont pour la plupart entre 14 et 20 ans, âge de la " crise d'adolescence ». (17) Comme le souligne Sour Marie de l'Immaculée, religieuse du Bon Pasteur d'Angers, c'est l'âge, pour tous les jeunes, filles ou garçons, de la " révolte juvénile [qui] est l'opposition plus ou moins brutale, envers tout ce qui peut gêner l'affirmation de soi, c'est-à-dire envers toutes les manifestations de la vie sociale ». ${ }^{(18)}$ Cependant, les jeunes filles placées ne sont pas des " adolescentes ordinaires ». Dans leur cas, plusieurs caractéristiques, telles que la dissociation et la faible valeur éducative du milieu familial, ou d'éventuelles " tares » intellectuelles ou caractérielles, ont " poussé la révolte juvénile [...] à se concrétiser dans le délit, la fugue, ou tout autre manifestation antisociale, à en faire au sens large du mot des "délinquantes" ».(19) Comme le résume Sœur Marie de l'Immaculée :

«De toutes ces considérations, il ressort qu'en ce qui concerne nos jeunes délinquantes, leur attitude d'agressivité est quelque chose de beaucoup plus profond que la crise de croissance que traversent toutes les adolescentes. Depuis l'enfance, elles sont sur la défensive, prêtes à l'attaque ou à la révolte. » ${ }^{(20)}$

Surtout, ces jeunes filles « difficiles » sont placées, pour la plupart, contre leur gré. La directrice de Brécourt insiste particulièrement sur cet aspect, en évoquant ainsi les buts de l'internat :
Vaucresson, Vaucresson, brochure, 1974, p. 19.

(15) Renseignements extraits du dossier d'information, dossier individuel de la mineure P 73, Brécourt, archives départementales du Val-d'Oise, 688 W 423. Tous les prénoms ont été modifiés.

(16) Dossier individuel de la mineure C 72 , Brécourt, archives départementales du Vald'Oise, 688 W 140.

(17) Sur l'émergence de cette notion dans la deuxième moitié du XIXème siècle, voir Agnès Thiercé, Histoire de l'adolescence (I850-I9I4), Paris, Belin, « Histoire de l'éducation », 1999, 359 p.

(18) Soeur Marie de l'Immaculée, La révolte juvénile, ses manifestations à l'accueil et à l'observation, 1949, p. 1, archives du Bon Pasteur d'Angers. 
(19) Ibid., p. 19

(20) Ibid., p. 24.

(21) « L'institution professionnelle d'éducation surveillée pour filles de 16 à 21 ans ", note interne, s. d., Centre des archives contemporaines, Fontainebleau, 91 0162-5.

(22) Michelle Perrot,

"Ouverture ", in

Christine Bard et al., [dir.], op. cit., p. 10.

(23) Andrée Algan, Enquête sur la rééducation en institution des filles délinquantes et inadaptées qui font l'objet d'une décision judiciaire, Vaucresson, CFRES, 1966, p. 28.

(24) Rapport annuel de la direction de l'Éducation surveillée, 1962, op. cit., p. 202.

(25) Michelle Perrot, op. cit., p. 11.

(26) Ibid, p. 10.
« Maintenir en internat pendant 365 jours sur 365... des filles grandes adolescentes, placées contre leur gré, après inadaptation sociale notoire ; obtenir une réinsertion sociale ultérieure ; par conséquent : malgré la contrainte et la privation de liberté, obtenir l'adhésion des mineures à la rééducation de longue durée (environ 3 ans) qui s'impose. " ${ }^{(21)}$

Comme toutes les internes, ces jeunes filles sont également soumises aux contraintes de la vie en collectivité - manque d'intimité, emploi du temps strict, etc. -, contraintes sans doute encore accentuées par les spécificités de l'internat de rééducation.

Que la violence des mineures placées apparaisse logique aux acteurs du secteur peut surprendre, non seulement parce que la majorité d'entre elles n'a pas été placée suite à des actes de violence, mais aussi, tout simplement, parce que ce sont des filles. La jeune fille, tout comme la femme adulte, est, en effet, traditionnellement perçue comme étant " "naturellement" douce, passive [et] d'instinct hostile à la violence ».(22) Or, ces stéréotypes s'effacent lorsqu'il s'agit de jeunes filles à rééduquer, comme si leur placement était le signe qu'elles n'étaient pas (ou plus) vraiment conformes à leur nature féminine. Elles semblent même être considérées comme " plus violentes que les garçons (qui s’attaquent moins souvent aux éducateurs ou aux objets, et se battent plutôt entre eux) ». ${ }^{(23)} \mathrm{Ce}$ constat est certainement à lier à l'idée que les filles accepteraient moins bien leur placement, parce qu'elles « sont plus jalouses de leur liberté que les garçons, et que les solutions d'autorité les heurtent facilement ».(24) Il convient, cependant, de se demander si cette impression ne correspond pas également à une différence entre les niveaux de violence jugés tolérables pour des filles, d'une part, et pour des garçons, d'autre part. De façon générale, la jeune fille violente paraît plus inquiétante que son équivalent masculin, tant son comportement semble en contradiction avec la supposée nature féminine et paraît être le "signe d'un dérèglement encore plus profond ». ${ }^{(25)}$ La sensibilité des acteurs de la rééducation est donc, sans doute, plus grande à l'égard de la violence des filles qu’à l'égard de celle des garçons, cette dernière pouvant même être considérée comme naturelle, comme " une caractéristique de la virilité ».(26)

Même si la violence des filles placées est attendue comme un mal logique, elle n'est pas souhaitable, ni même tolérable. Différents moyens sont donc mis en œuvre pour tenter de la prévenir et de la réguler. 


\subsection{Prévenir et réguler la violence}

Dans le contexte d'un renouvellement général des méthodes de rééducation en internat, plusieurs innovations - souvent inspirées par le scoutisme, le modèle anglais et l'Éducation nouvelle ${ }^{(27)}$ - ont notamment comme objectif la prévention d'éventuelles explosions de violence. Il en est ainsi de l'activité physique, de préférence en plein air. À Brécourt, par exemple, toutes les filles font de l'éducation physique - sports collectifs, athlétisme, natation, etc. - dans le vaste parc de la propriété. Quant aux plus turbulentes, elles travaillent dans la section horticulture, autrement dit à la ferme, ou encore pratiquent l'hébertisme une heure par jour. ${ }^{(28)}$ L'objectif du fondateur de cette " méthode naturelle » d'éducation physique, Georges Hébert - éducateur et ancien officier de marine -, était d'éduquer l'enfant tant du point de vue moral que du point de vue physique en lui faisant pratiquer, en plein air, les activités de l'homme à l'état primitif : marcher, courir, grimper, sauter, porter, lutter, etc. De plus, des efforts sont réalisés pour tenter d'atténuer les effets de l'enfermement : accueil d'intervenants extérieurs pour des concerts, par exemple -, sorties collectives, camps de vacances, nombre croissant de visites autorisées, permissions plus fréquentes dans la famille... Cependant, cette ouverture est progressive et, par exemple, à Brécourt en 1961, la sortie libre sans accompagnement n'est pas autorisée, contrairement à ce qui se fait dans les internats publics de garçons. ${ }^{(29)}$

Un autre mode de prévention repose sur l'occupation permanente des mineures placées, comme l'indiquent clairement la densité et le minutage des emplois du temps des internats. Selon la directrice de Brécourt, il y a lieu « d'astreindre les mineures à un travail assidu du matin au soir, tout en évitant lassitude ou révolte et ce, pour provoquer la fatigue physique, canaliser l'agressivité, sublimer les pulsions instinctuelles, "entraîner" à l'effort ". ${ }^{(30)}$ Cette activité permanente a également pour objectif de limiter les rêveries et l'introspection, afin d'éviter une éventuelle excitation à la violence par l'évocation du passé.

L'une des principales méthodes de prévention est, sans doute, la division de la collectivité pour en atténuer les inconvénients. Comme l'explique la directrice de Brécourt :

« Il y a lieu notamment de briser la collectivité en créant des groupes restreints : [...] 8-10 par groupe d'internat, 10-12 en atelier, 12-15 par classe d'enseignement général.

(27) Voir notamment :

Mathias Gardet et

Françoise Tétard, [dir.],

Le scoutisme et la rééduca-

tion dans l'immédiat

après-guerre, lune de miel

sans lendemain ?, Actes du

colloque de Vaucresson,

17-18 mars 1994, organi-

sé par le CNFE-PJJ, le

CRIV, l'AHES-PJM

et le CNAHES, document

de l'INJEP, n' 21,

juin 1995, 209 p. ; Agnès

Thiercé, op. cit.

(28) Voir par exemple le dossier individuel de la mineure S 70, Brécourt, archives départementales du Val-d'Oise, 688 W 479.

(29) « Le problème des sorties du dimanche dans les internats publics d'éducation surveillée ", s. d., Centre des archives contemporaines, Fontainebleau, 91 0300-48.

(30) « L'institution professionnelle d'éducation surveillée pour filles de 16 à 21 ans ", op. cit. 
(31) Ibid.

(32) Sœur Dublanc, op. cit., p. 12 .

(33) Ibid.
Buts :

a) Empêcher les scènes d'hystérie collective suivies d'incidents graves ;

b) Possibilité d'individualisation de l'action pédagogique ;

c) Pédagogie de groupe ${ }^{(31)}$

Cette volonté de diviser l'effectif en groupes de petite taille se retrouve progressivement dans tous les internats de rééducation de filles, à partir des années 1940. Les objectifs en sont multiples : mettre en place une action pédagogique plus individuelle, créer un cadre de vie familial, mais également prévenir la violence.

Il est une façon plus radicale de «briser la collectivité » : l'isolement, qu'il soit préventif ou curatif. Sœur Dublanc énumère « six cas où l'isolement est, pour ainsi dire, systématique " (32) au centre d'observation de Chevilly-Larue. Trois paraissent relever de la sanction : " la tentative de fugue », " une bagarre » et " un refus de travail », et trois de la prévention : « la trop grande excitation », « une fille trop tendue " qui s'intègre mal et contre laquelle le groupe se monte et, enfin, à la demande de la fille elle-même, par exemple si elle se sent trop "bouleversée ", énervée, ou bien si " elle manifeste le désir de travailler seule dans une pièce ". ${ }^{(33)}$ Lisolement est donc, dans certains cas, un traitement préventif, qui tente d'atténuer les inconvénients de la vie en collectivité en permettant à certaines d'avoir un peu de solitude, et surtout en évitant la contamination et la contagion.

La volonté de prévenir les explosions de violence a donc pour conséquence, peut-être inattendue, la mise en évidence de plusieurs limites de la rééducation en internat, ce qui entraîne de nombreuses évolutions et adaptations sans pour autant que soit réellement remis en cause le principe même de l'internat avant les années 1960. Il n’en reste pas moins que les grandes révoltes collectives ou " foires " paraissent relativement rares lorsque ces principes pédagogiques sont appliqués, ce qui est progressivement le cas dans tous les internats de rééducation. Ainsi, l'établissement de Brécourt, conçu dès son ouverture, en 1947, sur ce modèle, semble peu confronté à ce type de violence. Cependant, au moins une mineure, Madeleine, est précisément entrée dans cet établissement à la suite d'une révolte dont elle aurait été la meneuse au centre d'observation de Chevilly-Larue, où elle avait été placée au titre du vagabondage. Son parcours dans le secteur de la rééducation est ainsi synthétisé par la direction de Brécourt : 
"Après des fugues répétées en 1945, l'enfant, placée en dépôt au monastère de Chevilly-Larue, participe à la révolte du 28 octobre 1945. Confiée à la Tutélaire par ordonnance $[e n]$ décembre 1945, elle se signale par son caractère difficile et son mauvais esprit. En juin 1946, elle fomente une révolte au cours de laquelle elle se propose de s'évader ; découverte, elle est conduite de nouveau à Chevilly où elle tente de mettre ses projets à exécution ; la police est saisie de la tentative de révolte ( 15 bols et 6 soucoupes brisés), et [en] juillet 1946 [la mineure est confiée] au Bon-Pasteur de Montauban. Madeleine fait appel et attend à Fresnes [un] nouveau jugement. " (34)

Elle sera finalement confiée à l'établissement de Brécourt. Cette mineure " difficile » - face à laquelle les différents établissements fréquentés semblent n'avoir eu d'autre solution que l'intervention de la police suivie de la modification de garde, quitte à renvoyer la mineure à son établissement d'origine - reste finalement près de quatre ans à Brécourt.

Cependant, la diminution de ce type de violence n'implique pas la totale disparition de la violence dans les internats de rééducation. Demeurent, en effet, diverses manifestations de violence, certes moins spectaculaires, mais auxquelles l'institution doit également faire face.

\section{La violence dans l'internat}

\subsection{Violence des filles et réactions de l'internat}

Beaucoup plus fréquentes que les grandes révoltes collectives sont les manifestations de violence individuelle, qu'elles aient ou non une cible définie : les colères violentes, les bagarres et les détériorations de matériel - bris de carreaux ou de vaisselle - semblent relativement fréquentes. Dans ce dernier cas, l'emploi du temps de Brécourt, qui tient également lieu de règlement intérieur, précise que « les élèves doivent payer ce qu'elles cassent ». ${ }^{(35)}$ En revanche, les scènes violentes donnant lieu à des rapports conservés dans les dossiers individuels sont plus rares, mais ces quelques comptes rendus détaillés permettent de saisir non seulement les manifestations violentes auxquelles sont confrontées les éducatrices, mais aussi les réactions de l'institution. Ainsi, le comportement de Colette, dite "débile mentale " et placée pour vagabondage et suspicion d'inceste, donne lieu à plusieurs rapports détaillés. ${ }^{(36)}$ Par exemple, deux jours après avoir réintégré son équipe suite à un séjour à l'isolement, elle commence à
(34) Renseignements extraits du dossier d'information, dossier individuel de la mineure T 18, Brécourt, archives départementales du Val-d'Oise, 688 W 488.

(35) « Emploi du temps IPES de Brécourt ", 1967, Centre des archives contemporaines, Fontainebleau, 2000 0111-7.

(36) Dossier individuel de la mineure P 49, Brécourt, archives départementales du Vald'Oise, 688 W 414. 
(37) Cette mineure a une chambre individuelle du fait de son énurésie.

(38) Rapport d'incident, dossier individuel de la mineure P 49, Brécourt, archives départementales du Vald'Oise, 688 W 414. Les initiales des noms ont été modifiées.

(39) Pascale QuincyLefebvre, «Familles, institutions et déviances, Histoire de l'enfance difficile (1880-fin des années 1930) », Paris, Économica, 1997, p. 222.

(40) Rapport du 9 avril 1952, en réponse à la note du directeur de l'Éducation surveillée du 31 mars 1952, archives départementales $\mathrm{du}$ Val-d'Oise, 715 W 5. hurler dans le dortoir où ses camarades font la sieste, tout en refusant d'aller dans sa chambre individuelle à l'étage. ${ }^{(37)}$ Le rapport précise ensuite :

"Elle descend en courant. Priée de remonter, elle disparaît dans la cuisine où elle recommence à hurler. Les deux éducatrices obtiennent qu'elle monte jusqu'au premier étage. Elle refuse d'aller plus loin sous prétexte qu'on lui en veut, qu'on la maltraite, et qu'aucune autre élève ne subit pareille injustice, etc.

"Elle monte chercher sa couverture, mais au moment d'entrer dans sa chambre, elle se rend compte qu'elle va être enfermée et se dirige vers le grenier, hurle, crie et bouscule Melle M. qui lui barre le passage. [...] Elle répète qu'elle se plaindra à Madame la Directrice qui, seule, peut donner l'ordre de l'enfermer. Elle pique une rage et se réfugie en hurlant dans l'angle de l'escalier. Copieusement aspergée d'eau froide, elle menace son éducatrice de lui "renvoyer la bassine à la figure" et promet de se venger des éducatrices.

"Tandis que Melle M. descend demander l'aide de Monsieur E., Colette se précipite vers l'escalier et renvoie brutalement contre une poutre Melle K. qui essaie de lui barrer le passage, mais évite de recourir à la violence.

"Monsieur E. conseille à Colette de monter dans sa chambre, mais ne pouvant se faire obéir, il lui saisit le poignet et l'entraîne de force. " (38)

À l'essai d'isolement succède donc l'aspersion d'eau froide, ce qui rappelle la pratique, largement répandue dans les établissements de préservation et de correction d'avant-guerre, de la douche froide, supposée « fortifie $[r]$ les faibles et calme $[r]$ les nerveux ». ${ }^{(39)}$ Finalement, les éducatrices demandent de l'aide à un membre masculin du personnel de l'établissement, qui lui-même use, en dernier recours, d'une certaine forme de violence physique. Pour autant, rien ne semble capable de réellement calmer cette mineure qui s'évade de l'établissement six mois plus tard. Elle est reprise dès le lendemain, mais il est décidé qu'elle attendra une décision définitive à la maison d'arrêt de Pontoise. Après plusieurs échecs, la modification de garde apparaît donc comme la meilleure solution.

La violence directement dirigée contre une éducatrice est, sans doute, plus redoutée par les acteurs de la rééducation qu'une colère comme celle de Colette. En réponse à une note du directeur de l'Éducation surveillée concernant les agressions commises par les mineur(e)s placé(e)s en internat, la directrice de Brécourt précise que, "sans qu’il y ait eu agression à proprement parler, plu- 
sieurs pupilles se sont livrées dans le courant de l'année à des voies de fait sur la personne des éducatrices ". ${ }^{(40)}$ Sept cas sont ensuite rapportés pour la période allant d'août 1951 à janvier 1952, sans que soient précisées les sanctions prises : coups de pieds, coups de poings, griffures au visage, envoi d'ustensiles à la tête... Ce type de violence est également mentionné par Sœur M., religieuse du Bon Pasteur d'Angers, qui évoque même une agression préméditée par une jeune fille placée au Bon Pasteur de Charenton, au début des années 1960. La mineure s'en était pris à " une Sœur qui avait un certain âge et qui était venue aider un peu dans le centre [...] Alors la Sour, elle avait tout son voile et son bonnet rouges de sang. Elle l'avait assommée avec une carafe. [...] Elle l'avait attirée en lui disant : "Regardez les beaux dessins que j'ai faits!", sur son lit, étalés. Pendant que la Sour regarde, Tchuc! Alors c'était une fille qui venait de Fresnes, on a été obligé de la renvoyer à Fresnes ».(41) Même s'il s'agit d'une autre forme de violence, la réaction de l'institution semble également être, dans ce cas, une modification de garde.

La violence peut enfin être orientée contre soi-même : automutilations plus ou moins sévères et tentatives de suicide. Parmi les dossiers étudiés, deux rapportent explicitement des faits de cet ordre. Ainsi, Monique, entrée à Brécourt au début des années 1960, pour " vol, faux, usages de faux [et] fugues pour retrouver des garçons ", ${ }^{(42)}$ " est un cas très difficile, moins par son indiscipline que par son apathie, son désintérêt et tendances dépressives se traduisant par de subites impulsions de fugue ou des tentatives de suicide $»{ }^{(43)}$ Cette mineure tente de s'empoisonner avec des médicaments peu de temps après son arrivée, puis trois mois plus tard se "cisaill[ $e]$ le poignet et $[s e]$ jet $[t e]$ par la fenêtre ", ${ }^{(44)}$ avant d'être finalement remise à ses parents.

Le cas de Stella permet également de deviner la relative impuissance de l'institution face aux tendances dépressives de certaines élèves. Stella, entrée à Brécourt pour vagabondage, au début des années 1950 , s'évade deux semaines après son arrivée. Elle est réintégrée trois jours plus tard et placée « dans une chambre d'isolement » ${ }^{(45)}$ où elle tente de se suicider. Un rapport est alors rédigé :

«Elle ne semblait pas du tout déprimée et se vantait même de s'évader à nouveau dans une quinzaine de jours "pour passer les fêtes dehors". C'est lundi, à 13 heures, alors qu'aucun fait nouveau n'était intervenu, qu'elle a déclaré à une autre élève, Lucienne, isolée dans la chambre voisine, qu'elle en avait
(41) Entretien avec

Sœur M., religieuse du Bon Pasteur d'Angers, février 2004.

(42) Résumé du cas, dossier individuel de la mineure K 15 , Brécourt, archives départementales du Val-d'Oise, $688 \mathrm{~W} 295$.

(43) Lettre de la directrice de Brécourt au juge des enfants, dossier individuel de la mineure K 15, Brécourt, archives départementales du Val-d'Oise, 688 W 295.

(44) Rapport semestriel, dossier individuel de la mineure K 15 , Brécourt, archives départementales du Vald'Oise, 688 W 295.

(45) Rapport, dossier individuel de la mineure B 119, Brécourt, archives départementales du Vald'Oise, 688 W 83. 
(46) Ibid.

(47) Lettre de la mineure adressée à son frère et retenue dans le dossier, début des années 1950, dossier individuel de la mineure J 17, Brécourt, archives départementales du Val-d'Oise, 688 W 284.

(48) Sour Dublanc, op. cit., p. 13-14.

(49) « L'institution professionnelle d'éducation surveillée pour filles de 16 à 21 ans ", op. cit. assez et qu'elle allait se pendre. Lucienne alerta aussitôt par ses cris et Melle C., éducatrice-chef arriva immédiatement. [Elle] s'était suspendue à un barreau avec un morceau de sa couverture - elle avait déjà subi un début d'asphyxie. Des soins lui furent donnés par Melle C. puis par le médecin de l'établissement. Dans la soirée, elle avait une nouvelle crise et affirmait son désir de se suicider. [...] Elle avait déjà invoqué des idées de suicide antérieurement à sa fugue. " (46)

La mineure est transférée à l'hôpital et ne réintègre pas l'internat par la suite. Même si la mineure semble avoir mal supporté la mise en chambre d'isolement, en déduire que cette tentative de suicide résulte uniquement d'un rapport difficile à l'institution ou encore que c'est la réaction de l'institution suite à la fugue qui l'a provoquée serait bien hasardeux. Ce serait d'autant plus risqué que l'état psychologique de la mineure n'est connu qu'à travers les traces laissées dans son dossier et que les facteurs d'un tel passage à l'acte sont très probablement multiples. Comment savoir, en effet, quel aurait été le comportement de la mineure en l'absence de cette mise en isolement ? Il n'en reste pas moins que la mise en chambre d'isolement peut très certainement être ressentie par les mineures comme une violence, ainsi que permet de le supposer la description faite par Liliane, entrée à Brécourt quelques mois plus tard, dans une lettre à son frère, lettre par ailleurs plutôt favorable à l'établissement : "Tu sais ici il y a les mitards qui sont comme les véritables prisons, avec la même odeur. » ${ }^{(47)}$ Cet aspect carcéral est également décrit - puis justifié - par Sœur Dublanc dans son mémoire sur l'isolement dans l'établissement de Chevilly-Larue à la fin des années 1960 :

"La cellule mesure environ $3 \times 3$; elle est située au dernier étage de la maison. Les murs et le sol sont cimentés, une grande fenêtre, à hauteur d'homme, grillagée à l'extérieur, leur permet de voir ce qui se passe à l'extérieur. Aucun meuble. Un rectangle, dans lequel sont posées à même le sol une paillasse et des couvertures, est limité par une barre de ciment fixée au sol. Une porte en fer, surmontée d'une ouverture grillagée, donne sur un couloir.

"Certes, la cellule, lorsqu'on la voit pour la première fois, donne une impression de prison et l'on aurait tendance à dire que c'est inhumain pour des adolescentes. Cependant au bout d'un certain temps, on se rend compte qu'il serait difficile de concevoir la cellule autrement. En effet, les mineures y 
entrent le plus souvent dans un tel état de fureur que rien ne résisterait sous leurs mains. [...] La nudité et l'aspect sévère de la cellule deviennent une sécurité pour la mineure, pour le groupe et pour les cadres. »(48)

À travers ces quelques cas, il apparaît que, face à la violence de certaines mineures, l'institution est relativement désarmée et n'a guère d'autre moyen d'action que d'éloigner la jeune fille violente de ses camarades, soit en l'isolant, soit en demandant une modification de garde, afin d'éviter, ou du moins de limiter, la contagion et la " contamination des meilleures par les pires ». ${ }^{(49)}$

Ce repérage des différentes facettes de la violence en internat ainsi que des réactions de l'institution conduit à se demander si les facteurs de la violence mis en avant par les acteurs du secteur - " révolte juvénile ", troubles psychologiques et affectifs, placement contre leur gré, privation de liberté, enfermement, etc. - suffisent à expliquer entièrement cette violence des filles. Sans négliger l'importance de ces facteurs, cette violence semble pouvoir, parfois, être également comprise comme une forme de résistance à la logique même de l'institution et à son objectif, la rééducation, à l'instar de certaines révoltes dans les Reform schools canadiennes entre 1930 et $1960 . .^{(50)}$

\subsection{Les filles à rééduquer : des " êtres à modeler " (51)}

Afin de tester cette hypothèse de la violence comme acte de résistance, il est tout d'abord nécessaire de rappeler que l'objectif de la rééducation est, pour les filles comme pour les garçons, une transformation profonde. En effet, la notion de rééducation est intrinsèquement liée à la conviction qu'il est possible de transformer des cerveaux encore jeunes et donc perçus comme malléables, et que cela est plus pertinent et efficace que de sanctionner. Ainsi, le juge des enfants Jean Chazal dégage trois étapes dans la rééducation : le déconditionnement du jeune délinquant, son reconditionnement et sa personnalisation grâce à un cadre permettant l'épanouissement. ${ }^{(52)}$ Dans la même logique, la directrice de Brécourt qualifie les mineures qui lui sont confiées d' " êtres à modeler ". Cette conception n'est pas nouvelle - elle soustend déjà, au XIXème siècle, la prise en charge des mineurs " acquittés comme ayant agi sans discernement ", selon l'article 66 du Code pénal, et placés afin d'être éduqués - mais il semble qu'après guerre, dans une atmosphère optimiste de réforme, la foi dans la possibilité de changer en profon-
(50) Voir Tamara Myers et Joan Sangster, « Retorts, Runaways and Riots :

Patterns of Resistance in Canadian Reform Schools for Girls, 1930-1960 », Journal of Social History, vol. 34, n 3, Spring 2001, p. 669-697.

(51) Dominique Riehl, Observation et notation, compte rendu d'une conférence donnée lors de la première session de formation des éducateurs, au centre d'observation de Savigny-sur-

Orge, du 4 au 28 novembre 1947 , archives du centre d'exposition « Enfants en Justice » de Savigny-surOrge, sans cote.

(52) Jean Chazal, L'enfance délinquante, Paris, PUF, collection "Que sais-je?", 1953, p. 74. 
(53) L'évocation des prostituées, symboles des femmes "perdues", alors que cette conférence ne porte pas spécifiquement sur leur cas, n'est sans doute pas anodine. Dominique Riehl, L'éducatrice d'IPES, compte rendu d'une conférence donnée lors de la première session de formation des éducateurs, au centre d'observation de Savigny-surOrge, du 4 au 28 novembre 1947 , archives du centre d'exposition « Enfants en Justice " de Savigny-surOrge, sans cote.

(54) Dominique Riehl, Observation et notation, op. cit.

(55) Sour Malenfer, La révolte juvénile, 1959, p. 58 , archives du Bon Pasteur d'Angers. deur les mineurs est encore plus grande. Or, pour réaliser un tel objectif, une certaine violence psychologique et symbolique paraît nécessaire.

Une différence majeure entre les filles et les garçons placés en internat de rééducation réside dans la définition concrète du résultat à obtenir, chacun semblant devoir incarner au mieux le rôle social traditionnellement assigné à son sexe. Pour les filles, l'objectif est donc d'en faire des femmes respectables, parfaites épouses, mères de famille et maîtresses de maison, ce qui correspond, au moins dans une certaine mesure, à l'idéal proposé dans la plupart des institutions scolaires ou professionnelles de filles jusqu'aux années 1960. Pour autant, la façon dont sont perçues les jeunes filles placées à leur arrivée en maison de rééducation les distingue des autres jeunes filles. En effet, si une mineure est placée, c'est souvent, justement, parce qu'elle paraît s'éloigner de cet idéal et risque de devenir une jeune fille " perdue », moralement déchue. D’ailleurs, cet écart entre l'objectif à atteindre et l'appréciation de la situation initiale de la mineure est implicitement évoqué par Dominique Riehl, fondatrice de Brécourt, en conclusion de sa conférence sur l'éducatrice, en 1947 : "J'affirme que nos "méthodes" ont permis à d'anciennes prostituées de prendre conscience de ces vérités, conscience d'elles-mêmes et leur ont donné le désir sincère d'une vie droite jusqu'à la création d'un foyer régulier et stable. " (53)

Une telle transformation implique le recours à un certain nombre de méthodes qui, malgré les intentions des acteurs, sont de nature coercitive : placement, notation systématique, même dans le cadre de la "vie familiale " - Dominique Riehl compare les mauvaises notes à des "gifles de mères de famille " ${ }^{(54)}$ - ou encore, et peut-être tout particulièrement, volonté de marquer une rupture avec la vie passée. Cette rupture est triple : la mineure quitte son milieu de vie habituel, elle est souvent éloignée de sa région d'origine et, enfin, une prise de distance sur le plan moral est également recherchée. Il s'agit d'amener la mineure à prendre conscience des déficiences de son mode de vie antérieur et donc, souvent, de sa famille. En effet, même si la coopération avec les familles se développe progressivement, le regard porté sur cette dernière est souvent chargé de reproches. L'image de la mère apparaît particulièrement négative comme le laissent supposer aussi bien les propos d'une religieuse du Bon Pasteur : "si peu d'entre elles ont expérimenté ce qu'est une vraie maman soucieuse de ses devoirs et du bonheur des siens ! ", ${ }^{(55)}$ que ceux de 
Dominique Riehl à propos du lever des mineures : " réveil comme par mère de famille (convenable!) (en opposition avec hurlements et grossièretés dont elles ont en général l'habitude) ».(56)

Il s'agit donc de faire adhérer la mineure à des normes et des valeurs qui sont supposées être les seules valables, ou du moins les meilleures. Force est alors de constater que l'objectif de la rééducation implique le recours à une forme de violence, certes symbolique mais bien réelle. L'anecdote rapportée à propos de Claire montre que le regard porté sur cette violence par les mineures n'est pas nécessairement le même que celui de l'institution. En juillet 1948, il est écrit dans le cahier d'observation de Claire :

"A malheureusement été transférée entre 2 gendarmes. Le fait l'a laissée complètement froide. Elle a déclaré qu'il vaut mieux 2 gendarmes qu'une assistante sociale, qu'elle aurait refusé de faire le voyage avec une assistante. Attitude? " (57)

Peut-être est-ce là " une attitude » comme cela est suggéré, mais cela peut aussi être l'expression d'un rejet de la mission et du message des assistantes sociales, et par extension d'un rejet de l'entreprise de rééducation ? Cet écart perceptible entre la logique de l'institution et celle de certaines filles est d'autant moins négligeable que, comme le souligne François Dubet, " la violence ne peut être séparée de sa représentation et de son expérience subjective, du fait que tel ou tel acte est ou non vécu par celui qui le commet et par celui qui le subit, plus ou moins directement comme une violence ». ${ }^{(58)}$ Ainsi, ce qui est perçu comme une violence par l'institution peut ne pas être vécu comme tel par les mineures et inversement, l'importance de l' "expérience subjective » des acteurs étant encore accrue lorsqu'il ne s'agit pas de violence physique.

L'entreprise de rééducation peut donc impliquer, du point de vue des mineures, une certaine forme de violence, et ce malgré la disparition progressive de la violence proprement corporelle et physique. Cette dernière, en effet, semble de plus en plus exceptionnelle après la seconde guerre mondiale. Certes, des méthodes héritées, telles que la mise en isolement, restent en usage, mais les coups, les privations de nourriture ou autres brimades physiques ne sont pas compatibles avec l'esprit de réforme des années 1940 et sont proscrits, aussi bien dans les institutions privées que publiques. ${ }^{(59)}$ Il n'en reste pas moins nécessaire de se demander dans quelle mesure les jeunes filles
(56) Dominique Riehl, L'éducatrice d'IPES, op. cit.

(57) Cahier d'observation, 26 juillet 1948 , dossier individuel de la mineure B 123 ,

Brécourt, archives départementales du Vald'Oise, 688 W 84.

(58) François Dubet, op. cit., p. 9

(59) Pour approfondir cet aspect, il faudrait pouvoir préciser les modalités et la temporalité de cette disparition progressive de l'usage de la violence proprement physique, grâce à une comparaison détaillée entre les établissements du XIXème siècle et d'avantguerre, d'une part, ceux des Trente Glorieuses, d'autre part. Cependant, une telle comparaison n'est pas réalisable en l'état actuel des recherches et des connaissances, en particulier parce que la 
violence physique, lorsqu'elle est utilisée, laisse peu, voire pas de traces dans les archives des établissements, surtout lorsque l'usage en est théoriquement proscrit.

(60) « L'institution professionnelle d'éducation surveillée pour filles de 16 à 21 ans ", op. cit.

(61) «Emploi du temps IPES de Brécourt ", op. cit.

(62) Tamara Myers et Joan Sangster, op. cit., p. 674 .

(63) Voir supra.

(64) Voir Nicole Edelman, Les métamorphoses de l'hystérique, du début du XIXème siècle à la Grande Guerre, Paris, La découverte, collection "L'espace de l'histoire", 2003, 350 p. placées tentent de résister à la logique de l'institution et si cette résistance passe par la violence.

\subsection{La violence des filles : une forme de résistance ?}

Tout d'abord, même si beaucoup de mineures acceptent leur placement et profitent de la formation professionnelle qui leur est dispensée, le nombre d'évasions montre clairement qu' " obtenir l'adhésion des mineures à la rééducation de longue durée (environ 3 ans) qui s'impose " ${ }^{(60)}$ n'est pas toujours simple. Parmi les 69 filles du corpus étudié, 39, soit environ 56,5\%, ont tenté ou réussi une ou plusieurs fugues. Certes, certaines reviennent d'elles-mêmes rapidement et les facteurs qui influent sur le désir d'évasion sont multiples. Cependant, la fugue peut être comprise comme l'expression d'un certain rejet de la rééducation et donc comme une absence d'adhésion. De même, la passivité, l'opposition systématique, ou encore l'évocation du passé et, en particulier, de " souvenirs plus ou moins édifiants ", ${ }^{(61)}$ peuvent être considérées comme une contestation de l'entreprise de rééducation. Comme le soulignent Tamara Myers et Joan Sangster, parler de sexe est en soi une forme de résistance à la normativité de pureté et de chasteté qui est imposée. ${ }^{(62)}$

En ce qui concerne les actes de violence, un certain nombre peut, sans doute, être principalement expliqué par le mal-être, le désespoir ou par les troubles comportementaux des mineures. Dans cette logique, la directrice de Brécourt utilise l'expression "hystérie collective » ${ }^{(63)}$ pour qualifier certains comportements des mineures. La notion d' " hystérie » étant fortement connotée et inscrite dans un ensemble de stéréotypes sur la psychologie féminine, ${ }^{(64)}$ les manifestations de violence collective se trouvent ainsi réduites à des phénomènes incontrôlés et irrationnels relevant de la fragilité mentale et de la trop grande sensibilité féminines.

Cependant, il semble possible d'étayer l'hypothèse d'une lecture en termes d'actes de résistance, et ce, en particulier mais pas exclusivement, dans les cas des violences collectives, qui caractérisent surtout le début de la période étudiée. Bien plus, cette résistance paraît même parfois stratégique et témoigner d'une claire compréhension des mécanismes du secteur de la rééducation. Ainsi, Sœur Marie de l'Immaculée évoque, dans son étude sur la " révolte juvénile ", plusieurs cas de filles qui agissent avec violence, seule ou à plusieurs, explicitement en vue d'être envoyées à Fresnes, et qui souvent parviennent à ce résultat : 
" "Aller à Fresnes", c'est-à-dire en prison, paraît bizarre comme revendication. Mais voici à quoi cela correspond dans leur esprit : on met à Fresnes les filles “dures”, celles qu'on ne peut pas garder ailleurs. Alors il ne reste qu'une solution au juge : les libérer. " ${ }^{(65)}$

Cette explication proposée par Sœur Marie de l'Immaculée rejoint les études sur les Reform schools canadiennes : certaines révoltes avaient clairement pour objectif l'envoi en prison, afin d'être traitées en adultes dans une prison de femmes et non comme des jeunes filles ou des enfants. ${ }^{(66)}$ La violence est alors explicitement utilisée par les mineures pour contester leur désignation comme jeunes filles difficiles et inadaptées devant être placées dans une institution spécialisée. C'est toute l'entreprise de rééducation, méthode spécifique de prise en charge de mineur(e)s, qui est alors contestée.

Est-il également possible d'interpréter la violence contre soi comme un mode de résistance ? Il est évident que limiter l'explication à un seul facteur serait bien réducteur. Cependant, peut-être est-il possible, dans certains cas, de comprendre une tentative de suicide ou des automutilations non pas seulement comme un acte de désespoir, de masochisme ou d'autopunition, mais aussi comme une volonté de réappropriation de son corps, de contestation de l'enfermement et peut-être même comme une anticipation d'une modification de garde ou d'un envoi à l'hôpital.

Malgré les nombreux efforts des acteurs du secteur, la violence n'est donc pas absente des internats d'après-guerre, qu'elle soit exercée par les mineures ou ressentie par elles. Ce constat, alors que les différents acteurs du secteur ne cessent d'insister sur le caractère nécessaire et indispensable de l'adhésion des mineures, a certainement contribué à l'évolution des méthodes de rééducation, surtout à partir des années 1960, ce qui a transformé les modalités du problème de la violence en institution de filles. Tout d'abord, l'ouverture croissante des internats et l'importance progressivement accordée à la coopération avec les familles ont remis en cause l'idée d'une nécessaire rupture avec le milieu d'origine et le passé. Ensuite, le regard porté par la société sur les violences, notamment sexuelles, faites aux femmes, et en particulier aux femmes mineures, a évolué, ce qui a permis une reconnaissance croissante du statut de victime dans les cas d'inceste et de viol. Cette évolution, ainsi que l'importance de plus en plus
(65) Sœur Marie de l'Immaculée, op. cit., p. 38 .

(66) Tamara Myers et Joan Sangster, op. cit., p. 683. 
grande donnée à la prise en charge psychothérapeutique ont, sans doute, amélioré l'appréhension des problèmes des mineures et favorisé une meilleure prévention des manifestations violentes. Enfin, à la fin des années 1960, c'est l'ensemble du modèle de l'internat de rééducation qui se trouve contesté et remis en cause tandis que se développent, de plus en plus, les foyers de semi-liberté et l'action éducative en milieu ouvert.

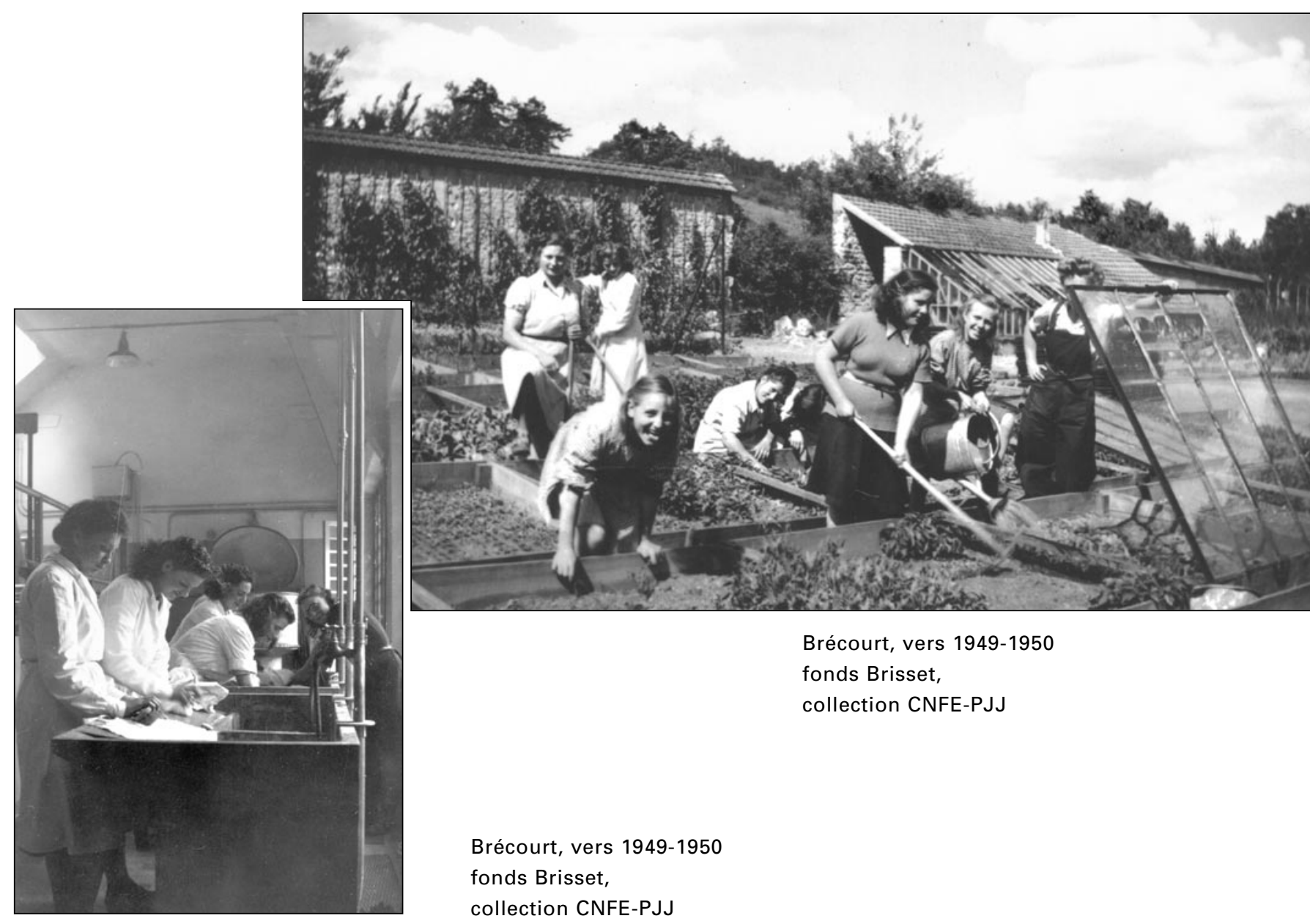


Cadillac, 1930

fonds Henri Manuel,

collection CNFE-PJJ

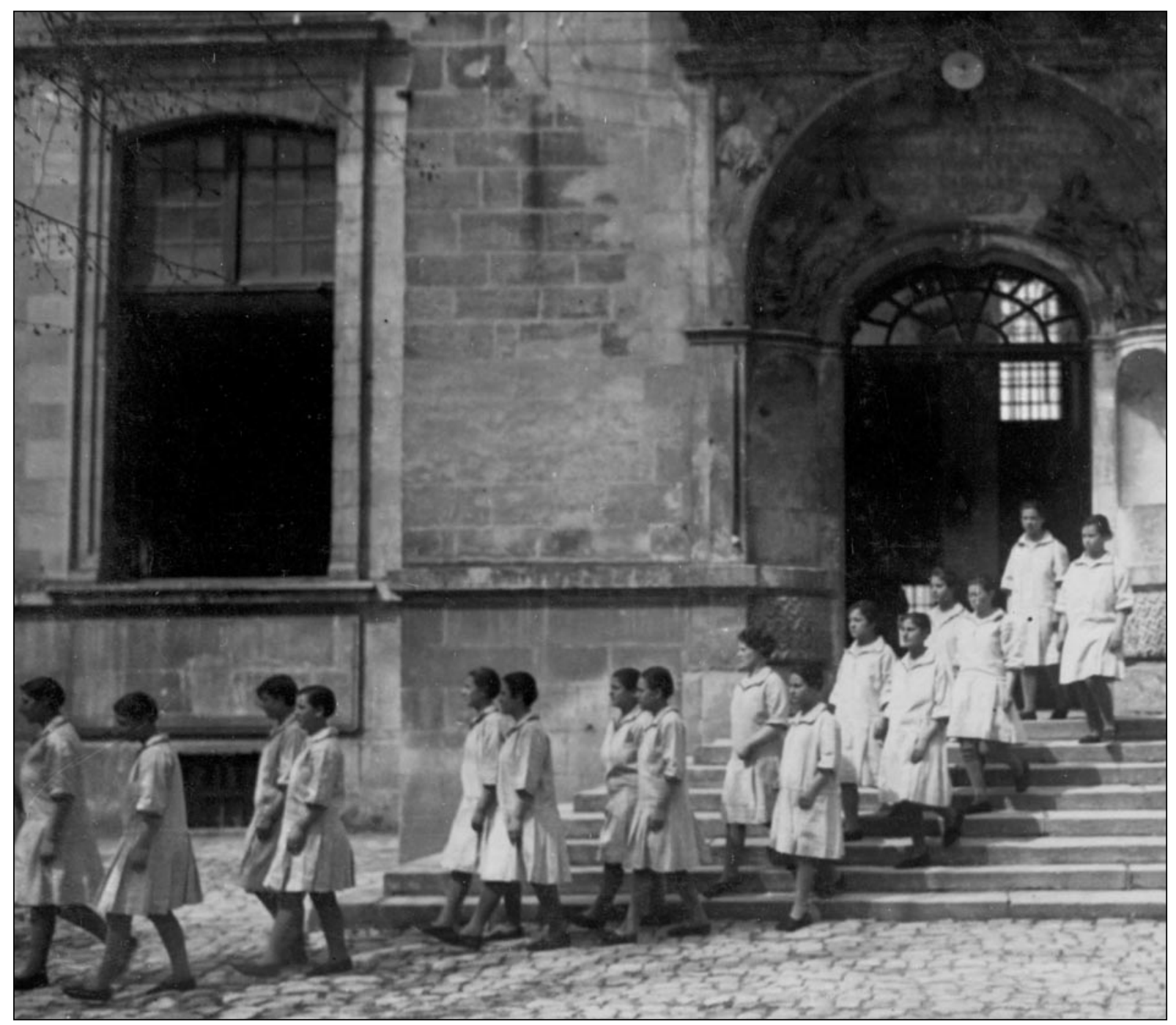

